\title{
Beef heart mitochondria for the Rotenone monitoring
}

\section{Eranda Mane, ${ }^{a}$ Sabrina Manente, ${ }^{b}$ Alessandra Iero, ${ }^{c}$ Silvia De Pieri, ${ }^{b}$ Gabriele Capodaglio, ${ }^{b}$ Francesca Cima, ${ }^{d}$ Loriano Ballarin ${ }^{d}$ and Marcantonio Bragadin $* b$}

\author{
Received 19th April 2010, Accepted 12th May 2010 \\ First published as an Advance Article on the web 28th May 2010 \\ DOI: 10.1039/c0ay00255k
}

\begin{abstract}
A new procedure for the selective monitoring of the Rotenone is proposed. Since the Rotenone inhibits the first site of the mitochondrial respiratory chain in all living organisms, the proposed method is based on measurements of inhibition of the respiratory rate of beef heart mitochondria.
\end{abstract}

Since its discovery, Rotenone has been used as insecticide, as fish poison to manage fish populations in reservoirs, lakes and streams, and as eliminating agent of undesirable species. This utilization is due to the toxicity of the compound which inhibits the first phosphorylating site in the mitochondrial respiratory chain. Since Rotenone has been largely used ${ }^{1,2}$ and is persistent, it can be an environmental problem.

As in the case of many other environmental contaminants, such as phenols, detergents, alkyl-metals and chloroanilines, the availability of biosensors for the monitoring, (in addition to the usual analytical technologies) is very convenient ${ }^{3-10}$ since a biosensor warranties low cost rapid measurements, and good reproducibility.

Since the action mechanism of Rotenone, which consists in an inhibition of the mitochondrial respiratory chain localized between NAD and the flavoprotein, ${ }^{11}$ is the same for all respiratory chains of all living organisms, we propose the utilization of the mitochondria from beef heart as biosensor for the selective monitoring of this compound. The mitochondria from beef hearth have been chosen because they are easily available from animals without any stabular. Moreover, the prepared mitochondria can be stored in freezer for months and can be utilized (in small doses) when necessary. After freezing, the mitochondria are generally no longer able to synthesize ATP, but only in the case of mitochondria from beef heart, the respiratory rate, which is sensitive to the presence of Rotenone, is not affected by freezing, so that they can be stored without losing efficiency.

All reagents for molecular biology were purchased from SigmaAldrich (Milan, Italy). MilliQ water was used.

The beef heart mitochondria were prepared following the procedure of Azzone et al. ${ }^{12}$

Once prepared, the mitochondria, subdivided in small amounts $(200 \mu \mathrm{l})$, were stored in freezer at $-20{ }^{\circ} \mathrm{C}$ and thawed before the measurements.

${ }^{a}$ Food Biotechnology Dept., Biotechnology and Feeding Faculty, Agricolture University,Tirana, Albania.E-mail: eranda.mane@ubt.edu.al ${ }^{b}$ Environmental Science Dept., Ca' Foscari University of Venice, Calle Larga S. Marta 2137, Dorsoduro, 30123 Venice, Italy. E-mail: bragadin@unive.it; Fax: +39041234 8584; Tel: +390412348507

'Institute of Applied Ecology, Health Science and Design, University of Camberra,Bruce, ACT, Australia.E-mail: aleaus2000@yahoo.au.com

${ }^{d}$ Biology Dept., Padua University, Via Ugo Bassi 58/, 35121 Padova, Italy
Mitochondrial protein concentration was determined by the Lowry method. ${ }^{13}$

The respiratory rate (the rate of oxygen consumption) was followed by means of a YSI 5331 Clark oxygen, a selective electrode in a thermostatted $\left(20^{\circ} \mathrm{C}\right)$ vessel $(2 \mathrm{ml})$. The $2.5 \mathrm{ml}$ Pyrex vessel, closed by a Teflon cap, was thermostatted at $20{ }^{\circ} \mathrm{C}$; the solution was magnetically stirred. Appropriate amounts of sucrose, $\mathrm{Mg}^{2+}$, buffer and mitochondria from a mother solution were added to the samples of water containing the Rotenone, in order to satisfy the standard operative conditions.

The inhibition of the respiratory chain (RC) by Rotenone takes place at the first phosphorylating site (Fig. 1).

The respiratory rate is the rate of oxygen consumption when a reducing substrate is added to the mitochondria. Fig. 2 shows a typical experiment when NADH is the reducing substrate.

The respiratory rate is constant since the reduction of the oxygen is linear with the time. The addition of Rotenone inhibits the respiratory rate. The graph in Fig. 2 correlates the respiratory rate with the Rotenone concentrations. As the respiratory rate depends on the amount of mitochondria, the graph in Fig. 2 illustrated the experiments performed at different protein concentration.

The results reported in Fig. 2 indicate that the affinity of Rotenone for its binding site is so high that the binding constant cannot be calculated.

The high affinity of Rotenone forward the binding site suggests the possibility of measuring very low quantities of Rotenone, using the mitochondria as a "trap" for the chemical compound.

In a typical experiment, the mitochondria $(0.8 \mathrm{mg})$ were re-suspended in the re-suspending medium $(50 \mathrm{ml})$ containing low doses of Rotenone $(1 \mathrm{nM})$. After centrifugation $(9000 \mathrm{~g} \times 10 \mathrm{~min})$, the mitochondria were re-suspended in a volume of $2 \mathrm{ml}$ of the same medium

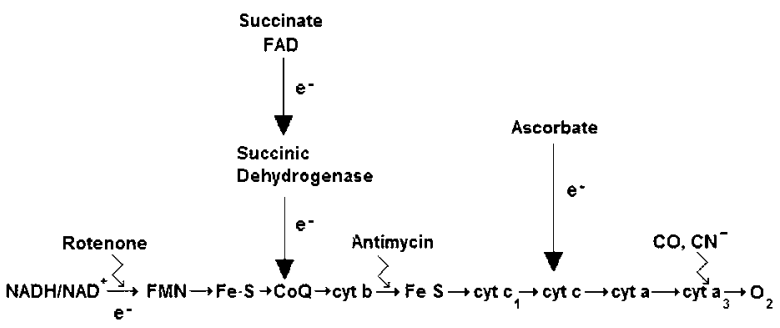

Fig. 1 The respiratory chain of mitochondria. In the mitochondria, a sequence of redox couples, the cytochromes, catalyzes the reduction of oxygen by substrates and ATP is produced. Three groups of substrates can be individuated, depending on their redox potential. NADH is the substrate with the highest redox potential. The respiratory chain is inhibited with selective inhibitors. Rotenone inhibits the first phosphorylating site. As a consequence, the reduction of oxygen by means of NADH is inhibited in the presence of Rotenone. 

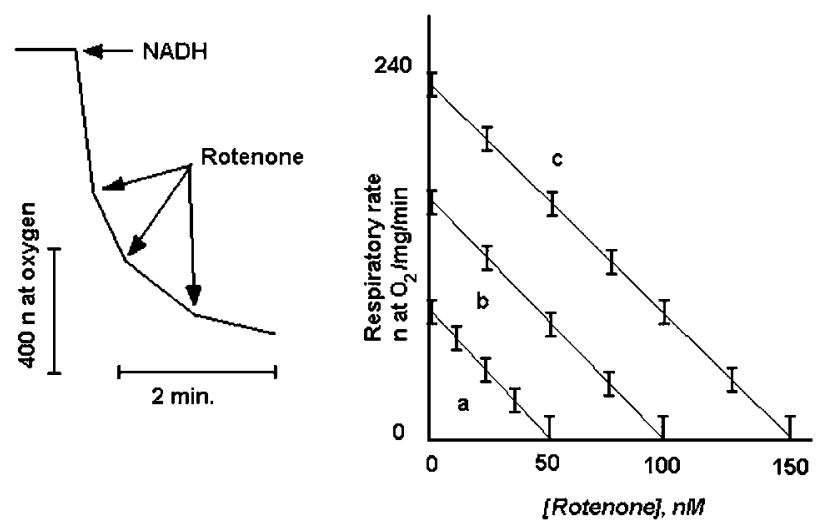

Fig. 2 A typical respiratory rate experiment in the mitochondria. The figure shows a typical experiment where the reducing substrate is NADH. The addition of Rotenone induces inhibition of the respiratory rate. The graph reports the respiratory rates obtained with successive additions of Rotenone and with varying amounts of mitochondria. Each point is the mean value of six experiments performed with two different mitochondrial preparations. Conditions: medium $0.25 \mathrm{M}$ sucrose (or $0.125 \mathrm{M} \mathrm{KCl}$ ), $10 \mathrm{mM}$ Hepes-Mops buffer $\mathrm{pH} 7.4,2 \mathrm{mM} \mathrm{MgCl} 2,5 \mathrm{mM}$ EDTA, $\%$ BSA (bovine serum albumin), $1 \mathrm{mM} \mathrm{FeSO}$. The arrows indicate the addition of $2 \mathrm{mM}$ NADH and Rotenone. Mitochondria: in (a) the concentration of thawed mitochondria was $0.4 \mathrm{mg} \mathrm{ml}^{-1}$ in (b) $0.8 \mathrm{mg} \mathrm{ml}^{-1}$, in (c) $1.2 \mathrm{mg}$ $\mathrm{ml}^{-1}$. After each measurements, the electrode was accurately and repetitively washed with a solution of $2 \%$ albumin.

and the respiratory rate compared with the rate of an analogous sample in the absence of Rotenone (blank). The mitochondrial concentration after the centrifugation and re-suspension was $0.4 \mathrm{mg}$ $\mathrm{ml}^{-1}$ in both samples and the respiratory rates were $40 \pm 6$ and $82 \pm 6$ nat oxygen $\mathrm{mg} \mathrm{min}^{-1}$ in the sample with Rotenone and in the blank respectively. In the graph of Fig. 2, the value of 40 corresponds to a $23 \pm 2 \mathrm{nM}$ concentration of Rotenone and, accounting for the dilution (25 times), this corresponds to the monitoring of $1 \mathrm{nM}$ Rotenone as effectively the initial concentration was.

As anticipated above, the respiratory rate of the mitochondria is conserved for months after freezing.

In some cases, however, a slight decrease of the respiratory rate is observed after a month period. This decrease is due to a cytochrome $c$ leak, and effectively, the maximal respiratory rate is restored after addition of $100 \mu \mathrm{M}$ of cytochrome $c$. The presence of cytochrome $c$ does not interfere with the measurements since it does not interfere with the Rotenone.

The procedure allows for the selective monitoring of the Rotenone even in the presence of other inhibitors of the RC for the following reasons:

- most inhibitors act on the succinic dehydrogenase (ref. 7) and the inhibition of this enzyme does not affect the oxidation of NADH (Fig. 1);

- the metals are good inhibitors of all step of the RC, but we have verified that in the presence of $5 \mathrm{mM}$ EDTA, a concentration of 1 $\mathrm{mM}$ metals (including $\mathrm{Cd}^{2+}$ and $\mathrm{Hg}^{2+}$, the most effective inhibitors) does not inhibit the RC. Therefore, taking into account that environmental samples do not contain such metal concentrations, we conclude that the metals are not a possible interference with the Rotenone inhibition.
Analogously, we have verified that $1 \%$ BSA protect against any possible interference due to the most common organic compounds, such as phenols, PCBs, chlorobenzene derivatives, benzofurans, atrazine derivatives and TCCD (tetrachlorodibenzo- $p$-dioxine) up to a concentration of $0.4 \mathrm{mM}^{6,7,10}$ Also in this case, it is to exclude that such categories of organic contaminants can interfere with the Rotenone inhibition, since generally the concentrations of these compounds do not exceed the micromolecular dose. Rotenone, like $\mathrm{NADH}$, is not removed by BSA because of the high affinity of the compound for the binding site.

The cyanide group can inhibit the $\mathrm{RC}$, but we have verified that in the presence of $1 \mathrm{mM} \mathrm{Fe}^{2+}$, as in our operative conditions, $0.5 \mathrm{mM}$ $\mathrm{CN}^{-}$(!) does not inhibit the RC. This is probably due to the fact that the $\mathrm{Fe}^{2+}$ is very high if compared to that of the iron in the mitochondrial cytochrome oxidase (Fig. 1) which could be inhibited by cyanide. In fact, in our conditions, the mitochondrial concentration is very low.

\section{Conclusions}

In conclusion, the proposed methodology offers the following advantages:

- high sensitivity and selectivity;

- availability and easy preparation of the mitochondria (from beef heart);

- low costs and rapid measurements.

The same procedure can be utilized for other environmental monitorings (detergents, organometals, phenols and whole toxicity in solution) (ref. 4,5,7 and 8). Taking into account the fact that about $200 \mathrm{~g}$ of mitochondria can be prepared from one beef heart and that each measurement requires some micrograms only, the procedure ensures the possibility of a relevant number of environmental monitorings. Moreover considering that each measurement requires less than $10 \mathrm{~min}$ and that the centrifugation of the samples requires about $10 \mathrm{~min}$, a complete test requires no more than $30 \mathrm{~min}$.

\section{Notes and references}

1 J. I. Fukami, I. Yamamoto and J. E. Casina, Science, 1967, 155, 713717.

2 P. E. Lindahl and K. EÖberg, Exp. Cell Res., 1961, 23, 228-232.

3 E. Argese, C. Bettiol, M. Fasolo, A. Zambon and F. Agnoli, Biochim. Biophys. Acta, 2002, 1558, 151-160.

4 M. Bragadin, E. Argese and E. Orsega, Environ. Technol., 1991, 12, 777-781.

5 M. Bragadin, D. Marton, A. Iero, S. Manente, G. Perin, V. Rizzoli and G. Scutari, Anal. Biochem., 1999, 269, 420-423.

6 G. A. Blondin, L. M. Knabeloch, H. W. Read and J. M. Arkin, Bull. Environ. Contam. Toxicol., 1987, 38, 467-474.

7 M. Bragadin and P. Dell'Antone, Arch. Environ. Contam. Toxicol., 1994, 27, 410-414.

8 M. Bragadin, R. Piazza, G. Scutari and S. Manente, Anal. Biochem., 2001, 292, 305-307.

9 B. I. Esher, M. Snozzi, K. Haberli and R. P. Schwarzenbach, Environ. Toxicol. Chem., 1997, 16, 405-414.

10 A. A. Bulich, Aquatic Toxicology Hazard Assessment ASTM STP, 1979, vol. 667, pp. 98-106.

11 K. EÖberg, Exp. Cell Res., 1961, 24, 163-165.

12 G. F. Azzone, R. Colonna and B. Ziche, Methods Enzymol., 1979, 55, 46-60.

13 O. H. Lowry, N. J. Rosenbrough, A. L. Farr and R. J. Randall, J. Biol. Chem., 1951, 193, 265-275. 